\title{
A GENERALIZED POINCARÉ INEQUALITY FOR GAUSSIAN MEASURES
}

\author{
WILLIAM BECKNER
}

(Communicated by J. Marshall Ash)

\begin{abstract}
New inequalities are obtained which interpolate in a sharp way between the Poincaré inequality and the logarithmic Sobolev inequality for both Gaussian measure and spherical surface measure.
\end{abstract}

The classical Poincare inequality provides an estimate for the first nontrivial eigenvalue of a positive self-adjoint operator that annihilates constants. For the Gaussian measure $d \mu=\prod_{k}(2 \pi)^{-1 / 2} e^{-(1 / 2) x_{k}^{2}} d x_{k}$ and the generator of the Ornstein-Uhlenbeck process $N=-\Delta+x \cdot \nabla$, the Poincare inequality is simply the estimate

$$
\int|f|^{2} d \mu-\left(\int f d \mu\right)^{2} \leq \int|\nabla f|^{2} d \mu .
$$

This is an old inequality in the folklore of Hermite polynomials and probably was known in one dimension to both mathematicians and physicists in the 1930 's in relation to eigenvalue problems ([9], [10]). It has been a useful tool in diverse subjects including partial differential equations [7] and statistics [4]. The purpose here is to note a generalized form of this estimate which interpolates in a sharp way between the Poincaré inequality and the logarithmic Sobolev inequality for Gaussian measures obtained by Gross [5].

Theorem 1. For $f \in L^{2}(d \mu), 1 \leq p \leq 2$ and $e^{-t}=\sqrt{p-1}$,

$$
\int|f|^{2} d \mu-\int\left|e^{-t N} f\right|^{2} d \mu \leq(2-p) \int|\nabla f|^{2} d \mu
$$

and

$$
\int|f|^{2} d \mu-\left(\int|f|^{p} d \mu\right)^{2 / p} \leq(2-p) \int|\nabla f|^{2} d \mu
$$

Such estimates are independent of dimension so one can regard the Gaussian measure space as infinite dimensional. The relation between these two

Received by the editors August 1, 1988.

1980 Mathematics Subject Classification (1985 Revision). Primary 42B99; Secondary 60D05.

This work was partially supported by the National Science Foundation. 
inequalities is provided by Nelson's hypercontractive estimates [8]

$$
\int\left|e^{-t N} f\right|^{2} d \mu \leq\left(\|f\|_{L^{p}(d \mu)}\right)^{2}
$$

when $e^{-t} \leq \sqrt{p-1}$. Inequality (2) gives the Poincare estimate at $p=1$ and inequality (3) implies the logarithmic Sobolev estimate

$$
\int|f|^{2} \ln |f| d \mu-\int|f|^{2} d \mu \ln \left[\int|f|^{2} d \mu\right]^{1 / 2} \leq \int|\nabla f|^{2} d \mu
$$

by a limiting argument as $p$ approaches 2 .

Since one can view Gaussian measure as the infinite-dimensional limit of normalized surface measure on a sphere ([6], [3]), similar estimates should hold for the sphere $S^{n}$. For $0<r<1$ the Poisson kernel on the sphere $S^{n}$ is defined by

with

$$
P_{r}(\xi, \eta)=\frac{1-r^{2}}{|\xi-r \eta|^{n+1}}
$$

$$
\left(P_{r} F\right)(\xi)=\int_{S^{n}} P_{r}(\xi, \eta) F(\eta) d \eta
$$

where $d \eta$ is normalized surface measure on $S^{n}$. The action of this kernel on spherical harmonics is given by

$$
\left(P_{r} F\right)(\xi)=\sum_{k=0}^{\infty} r^{k} Y_{k}(\xi)
$$

if $F$ has a development in spherical harmonics as $\sum Y_{k}$.

Theorem 2. Let $F \in L^{2}\left(S^{n}\right)$ and $u$ be the harmonic extension of $F$ to the interior of the unit ball in $\mathbf{R}^{n+1}$ with $\omega_{n}$ the surface area of the unit sphere $S^{n}$. For $1 \leq p \leq 2$ and $r=\sqrt{p-1}$,

(6) $\int_{S^{n}}|F|^{2} d \xi-\int_{S^{n}}\left|P_{r} F\right|^{2} d \xi \leq \frac{(2-p)}{\omega_{n}} \int_{|x| \leq 1}|\nabla u|^{2} d x \leq \frac{(2-p)}{n} \int_{S^{n}}|\nabla F|^{2} d \xi$ and

$\int_{S^{n}}|F|^{2} d \xi-\left(\int_{S^{n}}|F|^{p} d \xi\right)^{2 / p} \leq \frac{(2-p)}{\omega_{n}} \int_{|x| \leq 1}|\nabla u|^{2} d x \leq \frac{(2-p)}{n} \int_{S^{n}}|\nabla F|^{2} d \xi$.

The relation between inequalities (6) and (7) is given by the hypercontractive estimates for the Poisson semigroup obtained by the author in [3]

$$
\left\|P_{r} F\right\|_{L^{2}\left(S^{n}\right)} \leq\|F\|_{L^{p}\left(S^{n}\right)}
$$

when $0 \leq r \leq \sqrt{p-1}$.

The proofs of Theorems 1 and 2 follow by checking the action in inequalities (2) and (6) on the appropriate class of eigenfunctions. For a multi-index $\alpha$ of finite length $|\alpha|=\sum \alpha_{j}$, let $H_{\alpha}(x)=\prod H_{\alpha_{j}}\left(x_{j}\right)$ where the individual 
components of this product are Hermite polynomials of a single variable with the normalization

$$
e^{-t^{2} / 2+t y}=\sum_{l=0}^{\infty} \frac{t^{l}}{l !} H_{l}(y)
$$

Denote by $\pi_{k}(f)$ the projection of the function $f$ onto the linear span of $H_{\alpha}$ 's of length $|\alpha|=k$. Similarly a smooth function $F$ on the sphere $S^{n}$ will have a development in spherical harmonics of the form $F=\sum Y_{k}$. Then inequality (2) corresponds to the relation

$$
\int\left|\pi_{k}(f)\right|^{2} d \mu-(p-1)^{k} \int\left|\pi_{k}(f)\right|^{2} d \mu \leq(2-p) k \int\left[\left.\pi_{k}(f)\right|^{2} d \mu\right.
$$

and inequality (6) corresponds to the relation

$$
\begin{aligned}
\int\left|Y_{k}\right|^{2} d \xi-(p-1)^{k} \int\left|Y_{k}\right|^{2} d \xi & \leq(2-p) k \int\left|Y_{k}\right|^{2} d \xi \\
& \leq\left(\frac{2-p}{n}\right) k(k+n+1) \int\left|Y_{k}\right|^{2} d \xi
\end{aligned}
$$

Both of these inequalities are then determined by the simple relation

$$
1-(p-1)^{k} \leq(2-p) k
$$

for $1 \leq p \leq 2$, so if $p-1=1-\theta$ this follows from the elementary inequality $1 \leq(1-\theta)^{k}+k \theta$ for $0 \leq \theta \leq 1$ and $k \geq 0$. As noted above, inequalities (3) and (7) follow from (2) and (6) by using hypercontractive estimates.

Observe further that $1 \leq\left(1-(p-1)^{k}\right) /(2-p)$ for $k \geq 1$ and $1 \leq p \leq 2$; in fact, this ratio is monotone increasing in $p$ so that inequality (2) provides a smooth increasing interpolation for the Poincare inequality (1)

$$
\int|f|^{2} d \mu-\left(\int f d \mu\right)^{2} \leq\left[\frac{\int|f|^{2} d \mu-\int\left|e^{-t N} f\right|^{2} d \mu}{2-p}\right] \leq \int|\nabla f|^{2} d \mu
$$

where $e^{-t}=\sqrt{p-1}$.

The Poincare inequality appears similar to the "uncertainty principle" except that it is independent of dimension. Both inequalities can be obtained by considering the spectral resolution of a second-order selfadjoint differential operator acting on smooth functions in a Hilbert space. But a standard derivation of the uncertainty principle begins with the fact that for $f(x)=(2 \pi)^{n / 4} e^{x^{2} / 4} g(x)$

$$
0 \leq \int_{\mathbf{R}^{n}}|\nabla f|^{2} d \mu=\int_{\mathbf{R}^{n}}|\nabla g|^{2} d x+\frac{1}{4} \int_{\mathbf{R}^{n}}|x|^{2}|g|^{2} d x-\frac{n}{2} \int_{\mathbf{R}^{n}}|g|^{2} d x
$$

and then a dilation argument gives the usual form of the uncertainty inequality up to translation equivalence. With this perspective, one might view the Poincare inequality, the inequalities of Theorem 1 and the logarithmic Sobolev inequality as sharpened forms of the "uncertainty principle" on $\mathbf{R}^{n}$. 
Remarks. 1. Theorem 1 will follow from Theorem 2 by taking an infinitedimensional limit where the inequalities are rescaled to be on a sphere of radius $\sqrt{n}$ in $\mathbf{R}^{n+1}$ and recognizing in this limit that $-(1 / n) \Delta$ on $S^{n}$ will go to the generator of the Ornstein-Uhlenbeck process $N=-\Delta+x \cdot \nabla$. A similar calculation is discussed in [3].

2. The motivation for this note was the observation by the author that inequality (3) could be obtained as a consequence of the hypercontractive diffusion estimates of Bakry and Emery [1]. However, in Theorem 2 the generator of the Poisson semigroup is nonlocal and these estimates do not apply.

3. A proof of inequality (3) could also be given using two-point inequalities for Bernoulli trials as in the arguments of Gross [5] and the author [2]. In addition, convexity or Gaussian symmetrization [3] can be used to reduce this problem to the one-dimensional case.

4. Inequalities (2) and (6) can be reduced to the case of nonnegative functions by considering smooth functions and realizing that the form of the inequality is independent of additive constants since the measure space is finite.

\section{REFERENCES}

1. D. Bakry and M. Emery, Hypercontrativité de semi-groupes de diffusion, C. R. Acad. Sci. Paris 299 (1984), 775-778.

2. W. Beckner, Inequalities in Fourier analysis, Ann. Math. 102 (1975), 159-182.

3. W. Beckner, Sobolev inequalities, the Poisson semigroup and analysis on the sphere $S^{n}$, Proc. Nat. Acad. Sci. (to appear).

4. H. Chernoff, A note on an inequality involving the normal distribution, Ann. Probab. 9 (1981), 533-535.

5. L. Gross, Logarithmic Sobolev inequalities, Amer. J. Math. 97 (1975), 1061-1083.

6. H. P. McKean, Geometry of differential space, Ann. Probab. 1 (1973), 197-206.

7. J. Nash, Continuity of solutions of parabolic and elliptic equations, Amer. J. Math. 80 (1958), 931-954.

8. E. Nelson, The free Markoff field, J. Funct. Anal. 12 (1973), 211-227.

9. H. Weyl, The theory of groups and quantum mechanics, Dover Publications, New York, 1949.

10. N. Wiener, The Fourier integral and certain of its applications, Dover Publications, New York, 1958.

Department of Mathematics, University of Texas at Austin, Austin, TeXas 78713 